\title{
Unique Novel role of adropin in the gastric ulcer in a rotenone-induced rat model of Parkinson's disease
}

Rehab E. Abo El Gheit ${ }^{1}$, Marwa M. Atef ${ }^{2}$, Omnia S. El Deeb ${ }^{2}$, Ghada A. Badawi ${ }^{3}$, Hanan A. Alshenawy ${ }^{4}$, Walaa M. Elwan ${ }^{5}$, Heba M. Arakeep ${ }^{6}$, Marwa N. Emam ${ }^{1}$

${ }^{1}$ Physiology Department, Faculty of Medicine, Tanta University, Tanta, Egypt.

${ }^{2}$ Medical Biochemistry Department, Faculty of Medicine, Tanta University, Tanta, Egypt.

${ }^{3}$ Pharmacology and Toxicology Department, Faculty of Pharmacy and Pharmaceutical Industries, Sinai University, El-Arish, Egypt.

${ }^{4}$ Pathology Department, Faculty of Medicine, Tanta University, Egypt.

${ }^{5}$ Histology Department, Faculty of Medicine, Tanta University, Tanta, Egypt.

${ }^{6}$ Anatomy Department, Faculty of Medicine, Tanta University, Tanta, Egypt.

\section{Corresponding author:}

Rehab El sayed Abo El gheit

Assistant professor of physiology

Address: Physiology Department, Tanta University, El Geesh Street, Tanta, Egypt

Tel: $\quad+20 \quad(01276640441 \quad / \quad 01029595331)$

E-mail: $\quad$ rehab.abouelghait@med.TanTa.edu.eg

extrasystole.2003@gmail.com 


\section{Contents}

\section{Material and Methods;}

S.1. Macroscopic assessment of gastric mucosal damage;

S.2. Histopathological and immunohistochemical studies

S.2.1 Histological examination:

S.2.2 Immunohistochemical detection of SN p53 and gastric pro-angiogenic VEGF expression: S.2.2.1. Morphometric study:

\section{Results:}

S.3. Macroscopic assessment of gastric mucosal damage

S.5. Histological results of the stomach:

S.6. Immunohistochemical staining of striatal p53:

S.7.VEGF immunostaining of stomach:

S.8. Morphometric analysis of gastric VEGF, and striatal P-53 immunoreactivities in all studied groups

Table S1: Morphometric analysis of gastric VEGF and striatal P-53 immunreactivities in all studied groups.

Material and Methods; 


\section{S.1. Macroscopic Assessment of gastric mucosal damage;}

With the help of magnascope (under $5 \mathrm{X}$ amplification), mucosal lesions intensity and severity were scored and graded as following: no ulcer $=0$, epithelium shedding $=10$, petechial and frank haemorrhages $=20$, one or two ulcers $=30$, more than two ulcers $=40$ and perforated ulcers $=50$. The ulcer width and length $(\mathrm{mm})$ were measured under dissecting microscope $(1.8 \times)$ by a planimeter $\left(10 \times 10 \mathrm{~mm}^{2}=\right.$ ulcer area $)$. The ulcerated area was measured by counting the number of small squares, $(2 \times 2 \mathrm{~mm})$, covering the width and length of each ulcer band. The ulcer area (U, mm2) was calculated.

Ulcer area $(U)$ equal to the sum of small squares $\times 4 \times 1.8=U\left(\mathrm{~mm}^{2}\right)$

Percent protection index was calculated. ${ }^{42,43}$

$(\mathrm{Uc}-\mathrm{Ut})(\mathrm{UC})^{-1} \times 100$, where $\mathrm{Uc}=$ ulcer index in control group; $\mathrm{Ut}=$ ulcer index in treated group.

\section{S.2. Histopathological and immunohistochemical studies}

\section{S.2.1 Histological examination:}

Stomach and brain specimens were immersed in 10\% neutral-buffered formalin, washed, dehydrated, cleared and embedded in paraffin. Sections of $5 \mu \mathrm{m}$ thickness were stained with haematoxylin and eosin (H\&E).

\section{S.2.2 Immunohistochemical detection of SN p53 and gastric proangiogenic VEGF expression:}

Five $\mu \mathrm{m}$ thick gastric and brain sections were deparaffinized, rehydrated, and washed with phosphate buffered saline (PBS). Both sections were then incubated overnight in a humid chamber at $4^{\circ} \mathrm{C}$ with the primary antibody. For gastric sections, the primary antibody was rabbit polyclonal anti-VEGF (Santa Cruz Company, California, USA) (1: 500 in PBS). For brain sections, the primary antibody was rabbit polyclonal anti-p53 (Abcam, ab131442; Cambridge, Massachusetts, USA) (1: 100 in PBS). Both sections were then rinsed in PBS and incubated with the corresponding biotinylated secondary antibody for one hour at room temperature. Streptavidin peroxidase was then added for 30 minutes and rinsed again in PBS. Immunoreaction was visualized using 3,3'diaminobenzidine (DAB)-hydrogen peroxide as a chromogen. Sections were counterstained with Mayer's haematoxylin. The negative control sections were prepared by excluding the primary antibodies. The VEGF immunostained gastric sections were considered positive up on expressing brown cytoplasmic coloration. The p53 immunostained brain sections were considered positive upon expressing brown cytoplasmic and/or nuclear coloration. 


\section{S.2.2.1. Morphometric study:}

The images were examined using Leica Qwin $500 \mathrm{C}$ Image analyzer computer system (Leica Imaging System LTD., Cambridge, England) at (Central Research Lab, Faculty of Medicine, Tanta University, Egypt). Ten different non-overlapping randomlyselected fields from each slide were quantified for:

1. The mean color intensity of VEGF immunohistochemical positive reaction in DAB-stained gastric sections (At a magnification of 200).

2. The mean color intensity of $\mathrm{p} 53$ immunohistochemical positive reaction in DAB-stained brain sections (At a magnification of 400).

\section{Results:}

\section{S.3. Macroscopic Assessment of gastric mucosal damage}

As illustrated in (Figure 1), the macroscopic evaluation of gastric mucosa, both adropin and L-dopa/Om treatments resulted in significant reduction of the ulcer score compared to $\mathrm{PD} /$ Ulcer group with more evident gastro protective effect of adropin confirmed by significant decrease $(p<0.001)$ in the ulcer area that was reduced from $896.05 \pm 13.22$ $\mathrm{mm}^{2}$ in $\mathrm{PD} /$ Ulcer group to $371.15 \pm 9.37 \mathrm{~mm}^{2}$ in adropin treated group. Moreover, adropin displayed a higher protection of (84\%) compared to L-dopa/Om treated group that showed (76\%) protection.

\section{S.4. Histological results of the brain:}

As presented in (Figure 3), H\&E stained sections from control rats showed the SN formed of a sheet of closely packed neurons containing pale stained (vesicular) nuclei and deeply basophilic cytoplasm (Figure 3A). The PD/ Ulcer group II revealed marked neuronal damage of the $\mathrm{SN}$, where few neurons appeared and their nuclei were shrunken and deeply stained (pyknotic). Moreover, many vacuolar spaces were observed in the surrounding neuropil (Figure 3A). On the other hand, the adropin treated PD/ Ulcer (group III) revealed more or less normal neurons of SN (Figure 3A). While L-dopa/Om treated (group IV) showed lesser degree of neuronal injury as few cells appeared having pyknotic nuclei (Figure 3A).

\section{S.5. Histological results of the stomach:}

As presented in (Figure 4), H\&E stained sections from control (group I) showed the normal histological structure of fundus of stomach that was composed of mucosa, submucosa and muscularis externa. The gastric fundic mucosa was formed of 
epithelium, lamina propria and muscularis mucosa (Figure 4A). Group II (PK/Ulcer group) showed evident histological changes such as deep erosion of the gastric mucosa with presence of exfoliated dead cells in the lumen. Moreover, marked inflammatory cell infiltration, congested blood vessels were noticed in the lamina propria in addition to submucosal edema (Figure 4A). On the other hand, the adropin treated PD/ Ulcer group III showed complete restoration of the gastric mucosa that appeared intact and having normal structure. However, mild inflammatory cellular infiltration was noticed (Figure 4A). While in L-dopa/Om treated (group IV), partial restoration of the gastric mucosa was evident (Figure 4A).

\section{S.6. Immunohistochemical staining of striatal p53:}

Figure 3 revealed negligible p53- nuclear immunoreaction in the substantia nigra neurons of the control normal group I (Figure 3B). While sections from PD/ Ulcer group II revealed the degenerated neurons with strong positive p53 nuclear immunoreaction (Figure 3B). On the other hand, sections from adropin treated PD/ Ulcer (group III) showed faint positive p53 nuclear immunoreaction (Figure 3B). While sections from L-dopa/Om treated (group IV) showed weak positive p53 nuclear immunoreaction (Figure 3B).

\section{S.7.VEGF immunostaining of stomach:}

Figure 4 revealed that control group I showed a positive VEGF expression in the gastric mucosa that appeared as brown cytoplasmic reaction mainly in the wall of blood vessels (Figure 4B), whereas, the PD/ Ulcer group II revealed a weak positive cytoplasmic reaction of VEGF (Figure 4B). On the other hand, the adropin treated PD/ Ulcer (group III) showed a strong positive VEGF cytoplasmic reaction in the superficial and basal layers of gastric mucosa (Figure 4B). While the L-dopa/Om treated (group IV) showed a moderate positive cytoplasmic reaction of VEGF appeared first in the superficial layer of gastric mucosa (Figure 4B).

\section{S.8. Morphometric analysis of gastric VEGF, and striatal P-53 immunoreactivities in all studied groups}

The mean colour intensity of VEGF immunohistochemical reaction revealed a significant decrease in $\mathrm{PD} /$ Ulcer group compared with control group $(P<0.05)$, but it was significantly increased in both treated groups, compared with $\mathrm{PD} /$ Ulcer group $(P<0.05)$. Moreover, a significant difference was recorded between adropin and Ldopa/Om treated $\mathrm{PD} /$ Ulcer groups. As regards the mean colour intensity of p53 immunohistochemical reaction, it was significantly increased in PD/ Ulcer group 
compared with control normal group $(P<0.05)$, whereas it was highly significantly decreased in both treated groups, compared with PD/ Ulcer group $(P<0.05)$. Moreover, a significant difference was recorded between adropin and L-dopa/Om treated PD/ Ulcer groups, with better results in adropin treated animals (Table S1)

Table S1: Morphometric analysis of gastric VEGF and striatal P-53 immunreactivities in all studied groups.

\begin{tabular}{|c|c|c|c|c|}
\hline Mean color intensity / group & Normal control & PD/ Ulcer & Adropin treated & L-dopa/Om treated \\
\hline Gastric VEGF & $14.02 \pm 1.12$ & $10.09 \pm 1.23^{*}$ & $14.87 \pm 2.67^{\Delta \#}$ & $12.04 \pm 2.78^{\Delta}$ \\
\hline Striatal P-53 & $1.98 \pm 0.24$ & $26.87 \pm 5.16^{*}$ & $2.12 \pm 0.45^{\Delta \#}$ & $2.78 \pm 0.48^{\Delta}$ \\
\hline \multicolumn{5}{|c|}{$V E G F$ vascular endothelial growth factor. } \\
\hline \multicolumn{5}{|c|}{ * Mean significant difference vs control group $(P<0.05)$. } \\
\hline \multicolumn{5}{|c|}{${ }^{\Delta}$ Mean significant difference vs $\mathrm{PD} /$ Ulcer group $(P<0.05)$} \\
\hline \multicolumn{5}{|c|}{${ }^{\#}$ Mean significant difference vs L-dopa/Om treated group $(P<0.05)$. } \\
\hline
\end{tabular}

